\title{
Fabrication and Characterization of Electrospun Polycaprolactone Blended with Chitosan-Gelatin Complex Nanofibrous Mats
}

\author{
Yongfang Qian, Zhen Zhang, Laijiu Zheng, Ruoyuan Song, and Yuping Zhao \\ School of Textile and Material Engineering, Dalian Polytechnic University, Dalian, Liaoning, 116021, China \\ Correspondence should be addressed to Yongfang Qian; qyfdhu@126.com
}

Received 10 July 2014; Revised 18 August 2014; Accepted 21 August 2014; Published 2 September 2014

Academic Editor: Lan Xu

Copyright (C) 2014 Yongfang Qian et al. This is an open access article distributed under the Creative Commons Attribution License, which permits unrestricted use, distribution, and reproduction in any medium, provided the original work is properly cited.

\begin{abstract}
Design and fabrication of nanofibrous scaffolds should mimic the native extracellular matrix. This study is aimed at investigating electrospinning of polycaprolactone (PCL) blended with chitosan-gelatin complex. The morphologies were observed from scanning electron microscope. As-spun blended mats had thinner fibers than pure PCL. X-ray diffraction was used to analyze the degree of crystallinity. The intensity at two peaks at $2 \theta$ of $21^{\circ}$ and $23.5^{\circ}$ gradually decreased with the percentage of chitosan-gelatin complex increasing. Moreover, incorporation of the complex could obviously improve the hydrophilicity of as-spun blended mats. Mechanical properties of as-spun nanofibrous mats were also tested. The elongation at break of fibrous mats increased with the PCL content increasing and the ultimate tensile strength varied with different weight ratios. The as-spun mats had higher tensile strength when the weight ratio of PCL to CS-Gel was 75/25 compared to pure PCL. Both as-spun PCL scaffolds and PCL/CS-Gel scaffolds supported the proliferation of porcine iliac endothelial cells, and PCL/CS-Gel had better cell viability than pure PCL. Therefore, electrospun PCL/Chitosan-gelatin nanofibrous mats with weight ratio of 75/25 have better hydrophilicity mechanical properties, and cell proliferation and thus would be a promising candidate for tissue engineering scaffolds.
\end{abstract}

\section{Introduction}

Tissue engineering scaffolds play a key role that provides an environment for cells activities in regeneration of new tissue. In natural tissues, cells are surrounded by extracellular matrix (ECM), which is a complex composed of nanosized proteins and glycosaminoglycans (GAGs) [1-3]. Electrospinning has been proven to be a relatively simple technique that utilizes high-voltage electrostatic field to drive the polymer solutions or melts to produce nanofibers with diameter in the range from micrometers down to tens of nanometers, which has been investigated as a polymer processing technique for tissue engineering application in recent years $[4,5]$. Therefore, scaffolds fabricated by electrospinning could mimic the architecture of ECM.

Gelatin is a protein derived from partial hydrolysis of collagen, and chitosan from deacetylated chitin is a polysaccharide that has structure similar to GAGs in the ECM $[6,7]$. Therefore, electrospun chitosan and gelatin complex could further mimic the composition of natural ECM. During our previous work, chitosan and gelatin complex, defined as $\mathrm{CS} / \mathrm{Gel}$ below, was successfully electrospun into nanofibers [8]. However, the tensile strength and elongation of asspun mats were still too weak even after modification by cross-linking, which would limit its application as nanofibrous scaffolds. Thus, incorporation of a synthetic polymer would be necessary to improve the mechanical properties for tissue engineering applications. Desired physical properties could be achieved through the development of hybrid materials consist of synthetic and natural materials. Poly $(\varepsilon-$ caprolactone) (PCL) is a linear synthetic degradable polymer with high mechanical strength and biocompatibility [9] and is also a suitable candidate for fabricating fibrous scaffolds and has been approved by Food and Drug Administration (FDA) for various medical applications [10]. PCL has been blended with chitosan to improve the spinnability [11] and blended with collagen to fabricate aligned nanofibrous scaffolds to support the oriented growth of nerve axons [12]. However, 
electrospun PCL nanofibrous mats had poor hydrophilicity that would lead to reduction in its ability of cell adhesion, proliferation, and differentiation [13]. Therefore, hybrids made of synthetic and natural polymers could combine the mechanical properties of synthetic polymers and the biocompatibility of natural polymers. In the present work, PCL was blended with CS-Gel complex and then electrospun into nanofibrous mats. The fibers morphology, crystallites, wettability, and mechanical properties of as-spun mats were investigated by SEM, XRD, water contact angle test, and tensile measurement. To evaluate the application in tissue engineering, cell morphology and proliferation were also studied.

\section{Materials and Methods}

2.1. Materials. Chitosan $\left(\mathrm{M} \eta\right.$, about $\left.10^{6}\right)$ with a degree of deacetylation ( $\mathrm{DD}=85 \%$ ) was purchased from Haidebei Marine Bioengineering Co., Ltd. (Jinan, China). Gelatin (Type A, 300 bloom) and polycaprolactone (PCL) (Mw $=80000$ ) were purchased from Sigma-Aldrich (St. Louis, Missouri, USA). 1,1,1,3,3,3-Hexafluoro-2-propanol (HFP) was from Daikin Industries Ltd. (Japan) and trifluoroacetic acid (TFA) was from Runjie Chemical Reagent Company (Shanghai, China). All products were received without further purification.

\subsection{Preparation of the PCL Blended with CS-Gel Solutions and} Electrospinning. Chitosan and gelatin complex $(\mathrm{w} / \mathrm{w}=50 / 50)$ solution was obtained by mixing the chitosan solution, in which chitosan was dissolved in HFP/TFA (V/V = 8/2), and gelatin solution, in which gelatin was dissolved in HFP at the same volume ratio, with concentration of $0.08 \mathrm{~g} / \mathrm{mL}$ in the final mixed solution.

Polycaprolactone (PCL) was dissolved in pure HFP with concentration of $0.08 \mathrm{~g} / \mathrm{mL}$. PCL was blended with chitosangelatin complex at different weight ratios by mixing solutions at different volume ratios. Electrospinning was performed as previously described [8]. If brief, $1 \mathrm{~mL}$ plastic syringe containing the above mixed PCL/CS-Gel solutions was mounted in an accurate syringe pump (789100C, Cole-Parmer, USA). The mass flow rate was set to $1.0 \mathrm{~mL} / \mathrm{h}$. A voltage of $15 \mathrm{KV}$ between the syringe tip and the target, generated by highvoltage power supply (BGG DC high-voltage generator), purchased from the BMEI Co., Ltd. (Beijing, China), was applied to the tip of a syringe needle. The as-spun nanofibers were collected on a grounded metal plate, which was covered with aluminum foil (or had medical grade plastic coverslides onside) and placed $13 \mathrm{~cm}$ from the tip of the syringe needle. The electrospinning process was conducted under the ambient conditions.

\subsection{Characterization}

2.3.1. Scanning Electron Microscopy. The morphologies of the as-spun nanofibrous mats were observed by scanning electron microscope (SEM, JSM-5600LV, Japan) and the diameter distributions were conducted by image visualization software Image J (National Institutes of Health, USA).

2.3.2. Contact Angle Measurements. Surface wettability of the electrospun scaffolds was characterized by water contact angle measurement. The images of the droplet on the membrane were visualized through the image analyzer (OCA 40, Dataphysics, Germany) and the angles between the water droplet and the surface were measured. The measurement used distilled water as the reference liquid and it was automatically dropped on the electrospun scaffolds. To confirm the uniform distribution of blend nanofibrous scaffolds, the contact angle was measured three times from different positions and an average value was calculated by statistical method.

2.3.3. Mechanical Properties Test. The mechanical properties of the as-spun PCL/CS-Gel nanofibrous mats were tested using universal materials tester (H5K-S, Hounsfield, UK). All the samples $(50 \mathrm{~mm} \times 10 \mathrm{~mm})$ were prepared and tested in ambient temperature of $20^{\circ} \mathrm{C}$ and humidity of $65 \%$. The gauge length was $30 \mathrm{~mm}$ and the strain rate was set at $10 \mathrm{~mm} / \mathrm{min}$. Micrometer with precision of $0.01 \mathrm{~mm}$ was used to measure the thicknesses of the samples. The tensile stress-strain curves of the specimens were obtained from machine-recorded data.

2.4. Cell Viability and Proliferation. Porcine iliac endothelial cells (PIECs) were cultured in DMEM medium containing $10 \%$ fetal bovine serum, 100 units $/ \mathrm{mL}$ penicillin, and 100 units/mL streptomycin. Cells were placed in an incubator set to $37^{\circ} \mathrm{C}$ and $5 \%$ carbon dioxide $\left(\mathrm{CO}_{2}\right)$. The medium was changed every 3 days. Electrospun scaffolds were prepared on circular cover slips (14 $\mathrm{mm}$ in diameter). The cover slips were placed in 24-well plates and then secured with stainless Oring, sterilized with $75 \%$ alcohol solution which was replaced with phosphate-buffered saline solution (PBS) for washing after two hours.

To evaluate the morphology and proliferation of cells growing on the scaffolds, PIECs were seeded onto fibrous scaffolds and control glass cover slips. For the morphology study, PIECs were seeded carefully in the center of each well at a density of $1.0 \times 10^{5}$ cells $/ \mathrm{cm}^{2}$. After culturing for 24 hours, the cells on the mats were rinsed with PBS and then fixed in $4 \%$ glutaraldehyde water solution for $45 \mathrm{~min}$ at $4^{\circ} \mathrm{C}$ temperature. Thereafter, the samples were dehydrated in alcohol solutions with concentrations of $50 \%, 75 \%$, and $100 \%$ in turn and then dried under vacuum overnight. The dry cellular constructs were coated with gold sputter and observed under the SEM at a voltage of $10 \mathrm{KV}$. For cell proliferation tests, PIECs were seeded onto the as-spun mats at a density of $2.5 \times 10^{4}$ cells $/ \mathrm{cm}^{2}$. Cell proliferation was continually monitored on days $1,3,5$, and 7 after cell seeding. At the time points stated above, the cells and electrospun mats were incubated with $5 \mathrm{mg} / \mathrm{mL} 3$-[4,5-dimethyl-2-thiazolyl]2,5-diphenyl-2H-tetrazolium bromide (MTT) for $4 \mathrm{~h}$ and then the culture medium was extracted and $400 \mu \mathrm{L}$ dimethyl sulfoxide (DMSO) was added and stayed for 20 min. Finally, aliquots were pipetted into the wells of a 96-well plate and 
tested by an enzyme-labeled instrument (MK3, Thermo) and the absorbance at $492 \mathrm{~nm}$ for each well was measured.

\section{Results and Discussion}

3.1. Fiber Morphologies. Figure 1 shows SEM micrographs of electrospun mats prepared from PCL blended with CSGel complex at different weight ratios. From Figure 1(a), the as-spun pure PCL fibers lost their basic morphology of ultrathin fiber structure and interconnected between fibers. When the weight ratio of PCL to CS-Gel complex was $75 / 25$, the morphology of as-spun fibers became smooth and the nanofibers diameter decreased significantly from $893 \mathrm{~nm}$ to $114 \mathrm{~nm}$, as shown in Figure 1(b). The reason could be that the conductivity of the blended solutions was increased. Chitosan and gelatin have many polar groups, such as $-\mathrm{NH}_{2}$ and $\mathrm{COOH}$, that could carry positive or negative charges and form polyanion-polycation complex. The ejected jet would be subjected to greater elongation forces due to higher charge density on the surface $[14,15]$. Moreover, the jet bending instability could be enhanced because of the increasing of charge density and thus result in smaller fiber diameter $[16,17]$. However, when the weight ratio of PCL to CS-Gel complex was 50/50, the average diameter slightly increased to $154 \mathrm{~nm}$. The reason of increasing could be the diameters distributed at larger range from $40 \mathrm{~nm}$ to $388 \mathrm{~nm}$, while the diameters ranged from $57 \mathrm{~nm}$ to $221 \mathrm{~nm}$ when PCL content was $75 \%$. When estimating the size of as-spun diameters, at least 60 fibers randomly observed on the SEM images were calculated to get the average diameter. The proportions of fibers with diameters less than $100 \mathrm{~nm}$, ranging from $100 \mathrm{~nm}$ to $200 \mathrm{~nm}$, and more than $200 \mathrm{~nm}$ were $46.2 \%, 49.2 \%$, and $4.6 \%$, respectively, at PCL percentage of $75 \%$, while $40.3 \%$, $25.4 \%$, and $34.3 \%$, respectively, at PCL content $50 \%$. It could also be illustrated from Figure 1(c) that the fibers exhibit less uniform at PCL content of 50\% than fibers at PCL content of $25 \%$. For the CS-Gel complex, the average diameter was $231 \mathrm{~nm}$ and the diameters ranged from $63 \mathrm{~nm}$ to $707 \mathrm{~nm}$.

3.2. X-Ray Diffraction (XRD). Figure 2 shows $\mathrm{X}$-ray patterns of electrospun PCL blended with CS-Gel complex at different weight ratios. PCL showed a sharp peak at $2 \theta$ of $21^{\circ}$ and a relatively low intensity peak at $23.5^{\circ}$, showing the crystalline nature of PCL nanofibrous mats. With the percentage of CSGel complex increasing, the intensity of peak at $2 \theta$ of $21^{\circ}$ and $23.5^{\circ}$ gradually decreased and became amorphous broad peak for CS-Gel nanofibrous mats. The decreased intensity of blended nanofibrous mats indicates reduction in the degree of crystallinity and the as-spun CS-Gel mats have an amorphous structure.

3.3. Water Contact Angle Analysis. The surface wettability of biomaterials plays an important role for the attachment and proliferation of different cells $[13,18]$. To investigate the surface properties of fibrous mats, water contact angles were measured and shown in Figure 3. The pure PCL nanofibrous mats showed an angle around $128^{\circ}$ indicating that PCL nanofibrous scaffolds were hydrophobic. The reason could
TABLE 1: Mechanical properties of PCL blended with CS-Gel complex at different weight ratios $(n=3)$.

\begin{tabular}{lccc}
\hline PCL/CS-Gel & $\begin{array}{c}\text { Elongation at } \\
\text { break } \\
(\%)\end{array}$ & $\begin{array}{c}\text { Tensile } \\
\text { strength } \\
(\mathrm{MPa})\end{array}$ & $\begin{array}{c}\text { Thickness } \\
(\mathrm{mm})\end{array}$ \\
\hline $100 / 0$ & $127.2 \pm 12.22$ & $5.52 \pm 0.07$ & $0.119 \pm 0.015$ \\
$75 / 25$ & $85.87 \pm 2.41$ & $17.6 \pm 0.51$ & $0.096 \pm 0.005$ \\
$50 / 50$ & $34.93 \pm 5.88$ & $3.67 \pm 0.26$ & $0.111 \pm 0.014$ \\
$25 / 75$ & $19.47 \pm 2.34$ & $2.24 \pm 0.35$ & $0.201 \pm 0.017$ \\
$0 / 100$ & $2.71 \pm 0.16$ & $2.07 \pm 0.18$ & $0.106 \pm 0.005$ \\
\hline
\end{tabular}

be the presence of $\mathrm{CH}_{2}$ groups in the main chain of PCL that leads to the hydrophobic property. The contact angles of blended nanofibrous mats decreased to $69^{\circ}, 60^{\circ}$, and $28^{\circ}$, respectively, when the percentages of CS-Gel were $25 \%$, $50 \%$, and $75 \%$, implying that the blended nanofibrous mats transformed to be hydrophilic. The reason is that CS-Gel complex has many hydrophilic groups, such as amine and carboxyl. Hydrophilic surfaces exhibit better affinity for cells than hydrophobic surfaces [19]. However, when the CS-Gel content was more than $50 \%$, the membrane partly dissolved during the water contact angle test, and CS-Gel membrane dissolved completely.

3.4. Mechanical Properties of Electrospun PCL Blended with Chitosan-Gelatin Complex. The mechanical properties of asspun mats have an important effect on successful application in tissue engineering. The typical tensile stress-strain curves of PCL blended with CS-Gel complex nanofibrous mats were shown in Figure 4 . The average elongation at break and average ultimate tensile strength, as determined by the stress at break normalized to the cross-sectional area of asspun mats, were summarized in Table 1 . Figure 4 and Table 1 showed that the elongation at break of nanofibrous mats increased with the PCL content increasing, while the ultimate tensile strength varied with different weight ratios. The failure process of nanofibrous mats involved slippage and break of fibers. The unit of PCL molecule has five methylenes that lead to the good flexibility. When the percentage of CS-Gel complex was more than $50 \%$, as-spun nanofibrous mats exhibit typical brittle fracture. With increasing the PCL percentage to $75 \%$, nanofibrous mats transformed from brittle to flexible. However, it is interesting to find that pure PCL mats had lower tensile strength than PCL/CS-Gel (75/25). Lee et al. [2] studied the effect of gelatin incorporation into PLCL and also found that gelatin incorporation of $10-30 \mathrm{wt} \%$ had an enhanced tensile strength. The reason could be the reduction of fibers diameter when incorporating chitosan and gelatin complex. Fibers with thinner diameter would have better tensile strength. Thus, the mechanical properties of PCL/CSGel at weight ratio $75 / 25$ were better compared with both PCL and CS-Gel complex.

3.5. Morphology and Proliferation of PIECs on PCL and PCL/CS-Gel Scaffolds. When the weight ratio of PCL to 


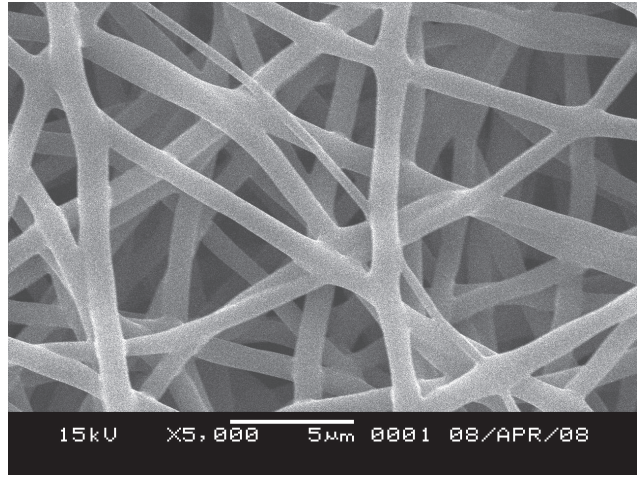

(a)

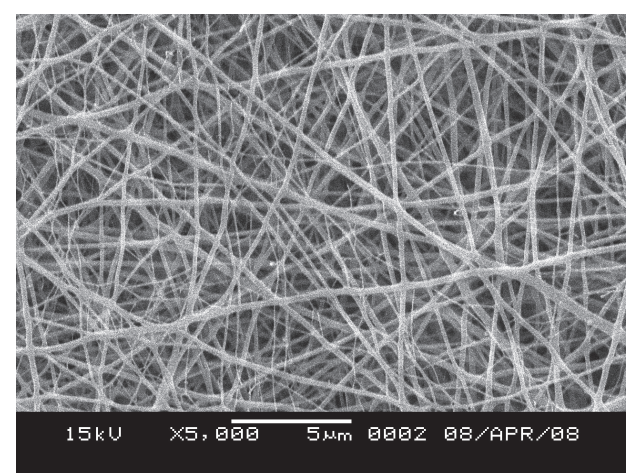

(b)

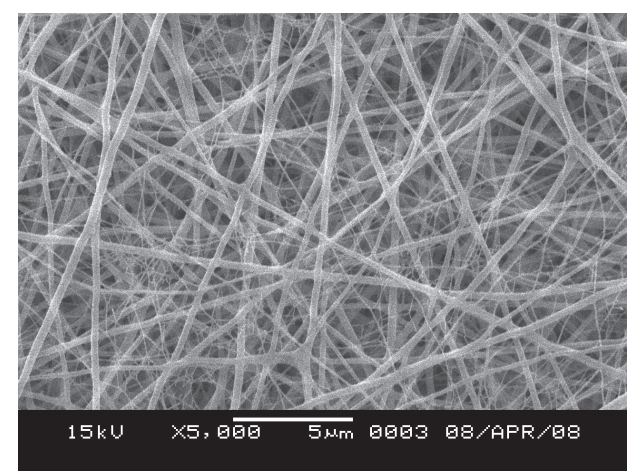

(c)

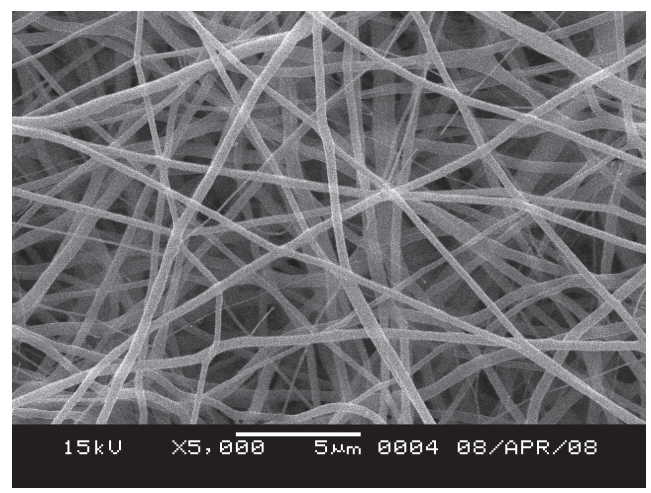

(d)

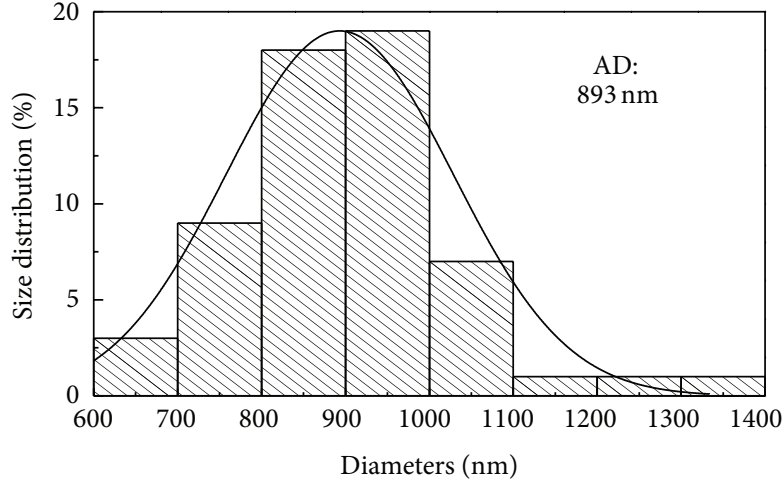

$\left(a^{\prime}\right)$

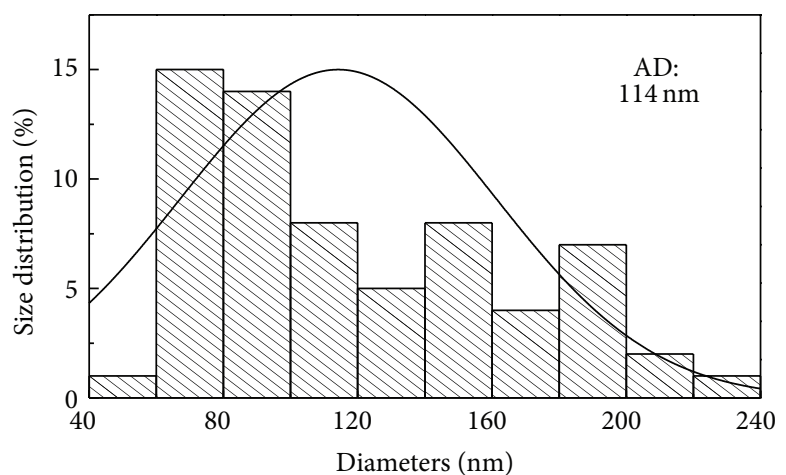

$\left(b^{\prime}\right)$

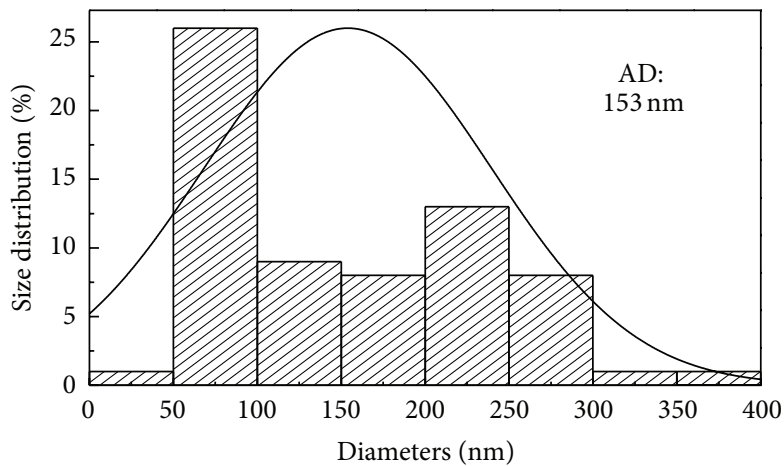

$\left(c^{\prime}\right)$

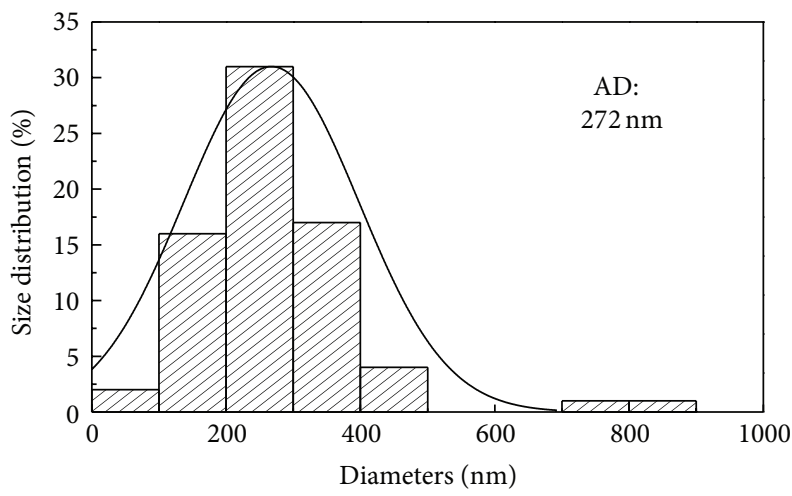

$\left(d^{\prime}\right)$

FIgURE 1: Continued. 


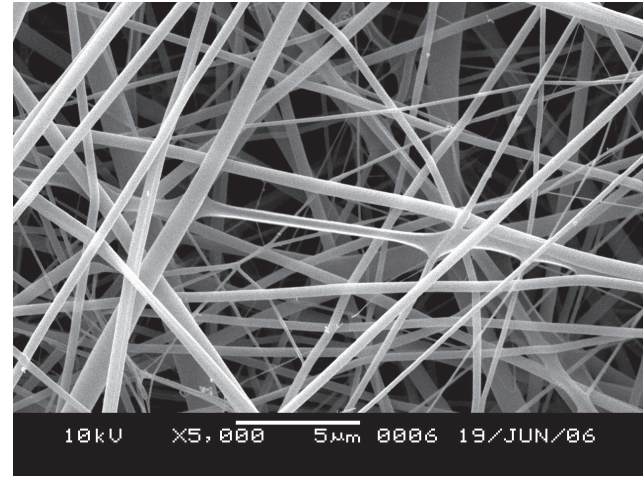

(e)

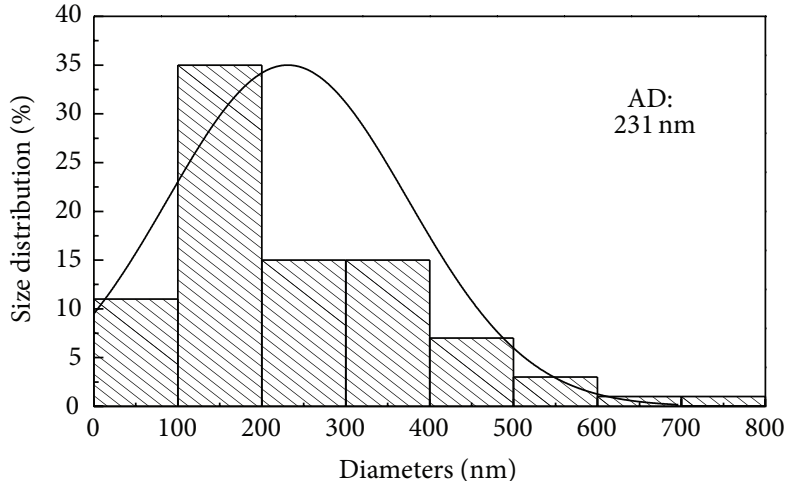

$\left(\mathrm{e}^{\prime}\right)$

FIGURE 1: SEM micrographs and diameter distributions of as-spun PCL blended with CS-Gel complex at different weight ratios, PCL to CS-Gel complex: (a) 100/0; (b) 75/25; (c) 50/50; (d) 25/75; (e) 0/100.

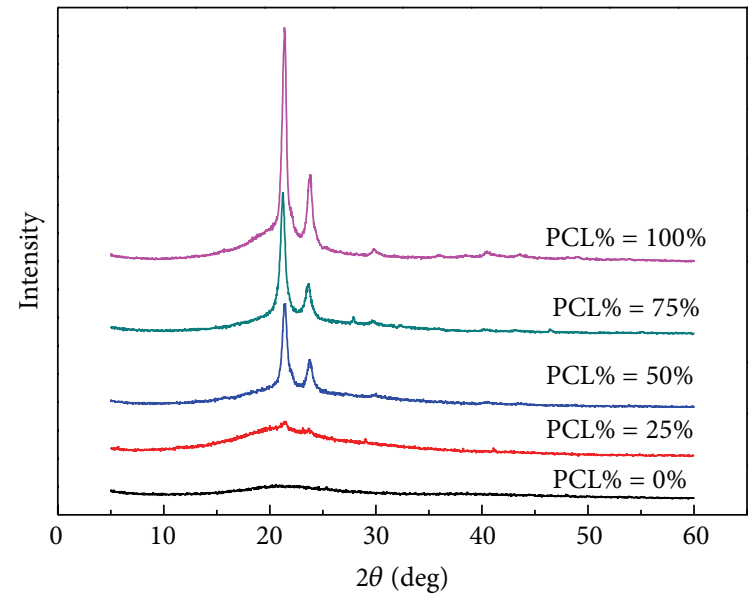

FIGURE 2: XRD pattern of as-spun PCL blended with CS-Gel complex nanofibrous mats with different percentages of PCL.

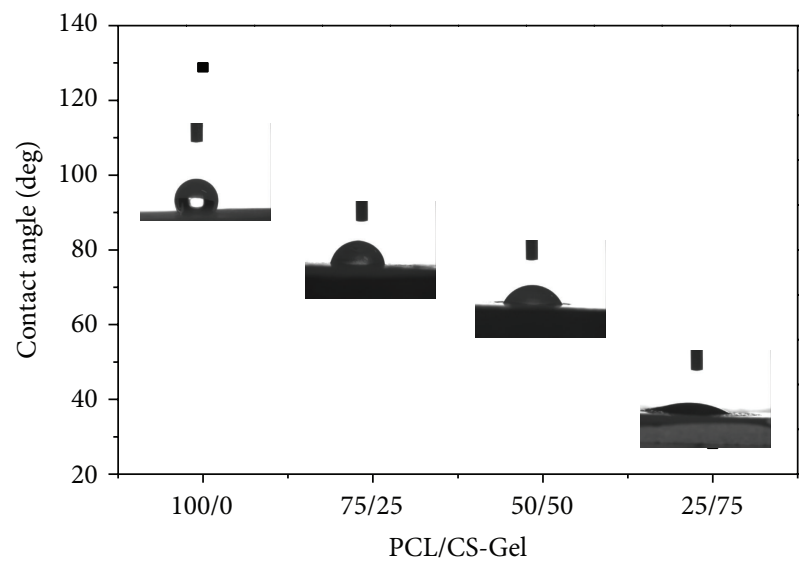

FIGURE 3: Water contact angle of electrospun PCL blended with gelatin and chitosan complex at different weight ratios (Inset of this figure shows the variation shapes of contact angle on different mats).

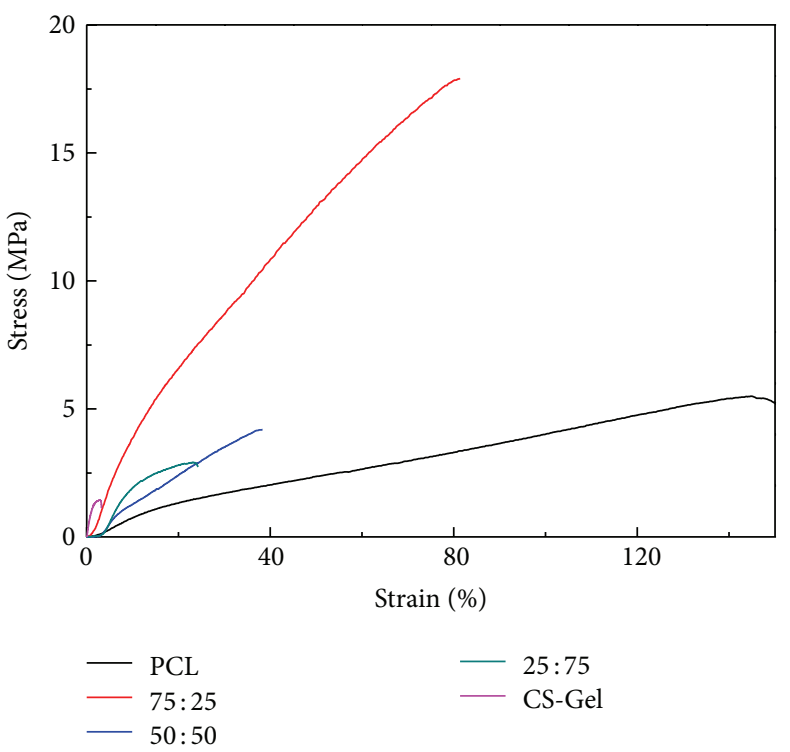

FIGURE 4: Mechanical properties of electrospun PCL blended with chitosan and gelatin complex at different weight ratios.

CS-Gel was 75/25, defined as PCL/CS-Gel below, the asspun mats had better mechanical properties and hydrophilic surfaces. The morphology of the cells cultured on PCL and PCL/CS-Gel scaffolds was evaluated by SEM. Figure 5 shows the cell morphology on PCL, PCL/CS-Gel scaffolds, and cover slips (control) at $24 \mathrm{~h}$ after seeding. PIECs extended well on the surface of the above two scaffolds with polygonal shape, and nanofibrous scaffolds had better cell viability in comparison with cover slips. Moreover, cells spread more easily to form endothelial layer on PCL/CS-Gel scaffold than pure PCL, implying that incorporation of CS-Gel into PCL could improve the biocompatibility. The incorporation of functional groups such as $\mathrm{NH}_{2}, \mathrm{COOH}$, and $\mathrm{OH}$ could introduce cell recognition sites to promote cell-material interactions [20]. 


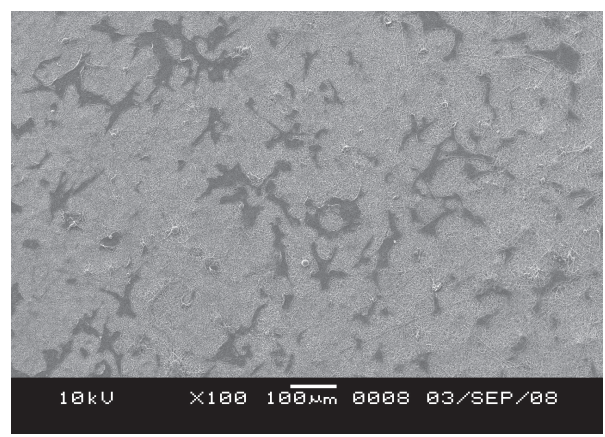

(a)

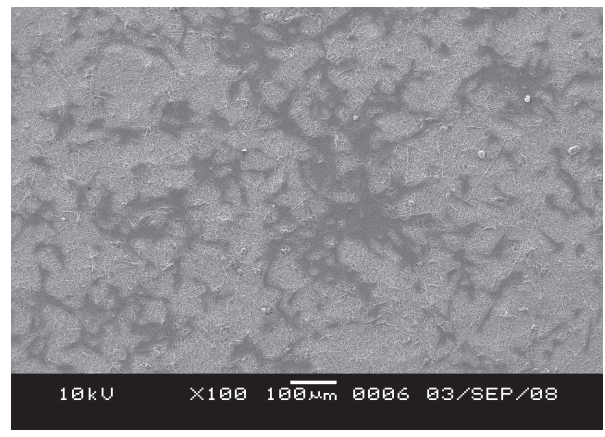

(b)

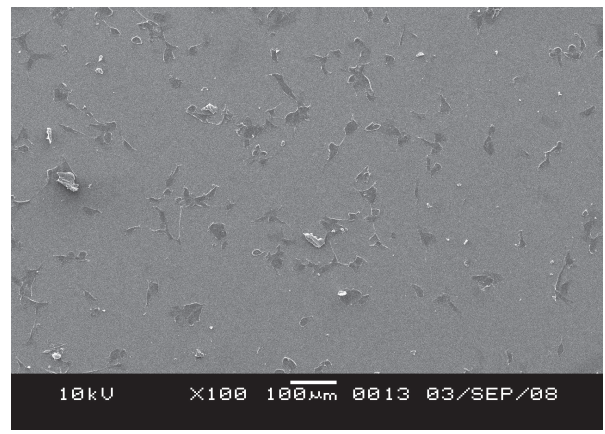

(c)

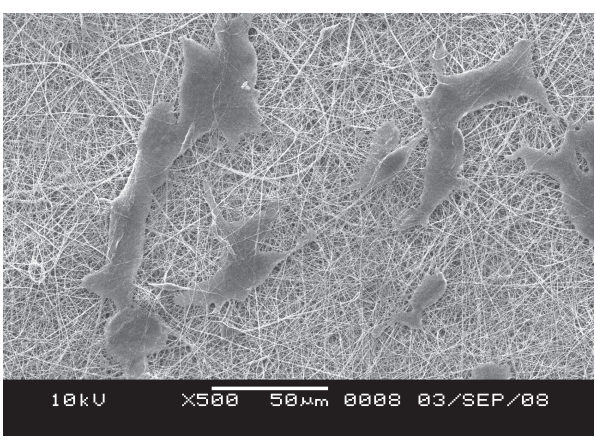

$\left(\mathrm{a}^{\prime}\right)$

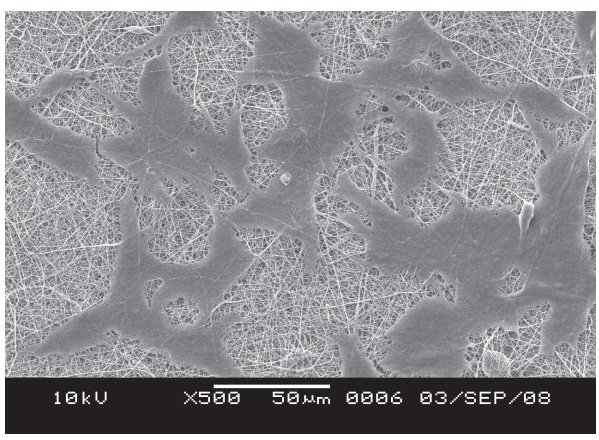

$\left(b^{\prime}\right)$

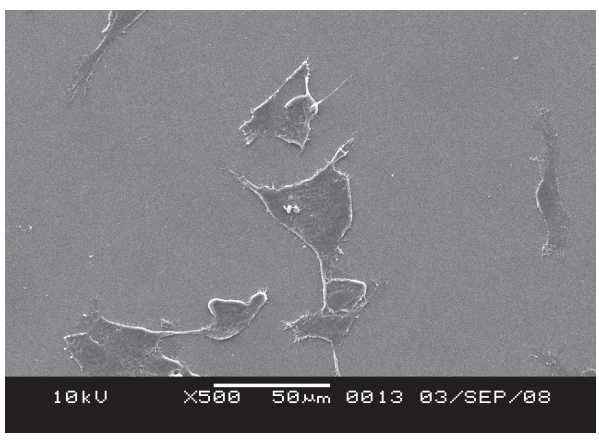

$\left(c^{\prime}\right)$

FIgure 5: Morphology of PIECs cultured on PCL, PCL/CS-Gel, and cover slips (control): (a) PCL, (b) PCL/CS-Gel, and (c) cover slips.

The proliferation of PIECs cultured on PCL, PCL/CS-Gel, cover slips, and tissue culture plates (TCPs) on days 1,3, 5, and 7 after seeding was shown in Figure 6. During being cultured for 7 days, cells grew well and exhibited increasing trend. On day 7, cells on PCL/CS-Gel appeared more increasing than PCL and cover slips. Thus, PCL/CS-Gel scaffolds would be potential candidate in tissue engineering.

\section{Conclusion}

In this work, we developed PCL blended with CS-Gel complex nanofibrous mats. PCL blended with CS-Gel complex at different weight ratios was successfully electrospun and subsequently characterized by SEM, XRD, and water contact angle test. SEM analysis indicates that blended mats have thinner fibers than pure PCL, and the average diameter was the smallest when PCL to CS-Gel (w/w) ratio was 75/25. From XRD analysis, the degree of crystallinity decreased with the percentage of CS-Gel complex increasing. Incorporation of chitosan-gelatin complex could obviously improve the hydrophilicity of as-spun mats from water contact angle test. However, the nanofibrous mats became partly dissolved when CS-Gel complex content was more than 50\%. From mechanical properties test, the elongation at break increased with the PCL content increasing. PCL/CS-Gel $(\mathrm{w} / \mathrm{w}=75 / 25)$ exhibited higher tensile strength. Finally, cell morphology and proliferation were studied. PCL/CS-Gel scaffolds have better proliferation than pure PCL. Based on these results, PCL/CS-Gel nanofibrous scaffolds with weight ratio of 75/25 have better hydrophilic property, mechanical properties, and cell proliferation, which would be a promising candidate for tissue engineering scaffolds. 


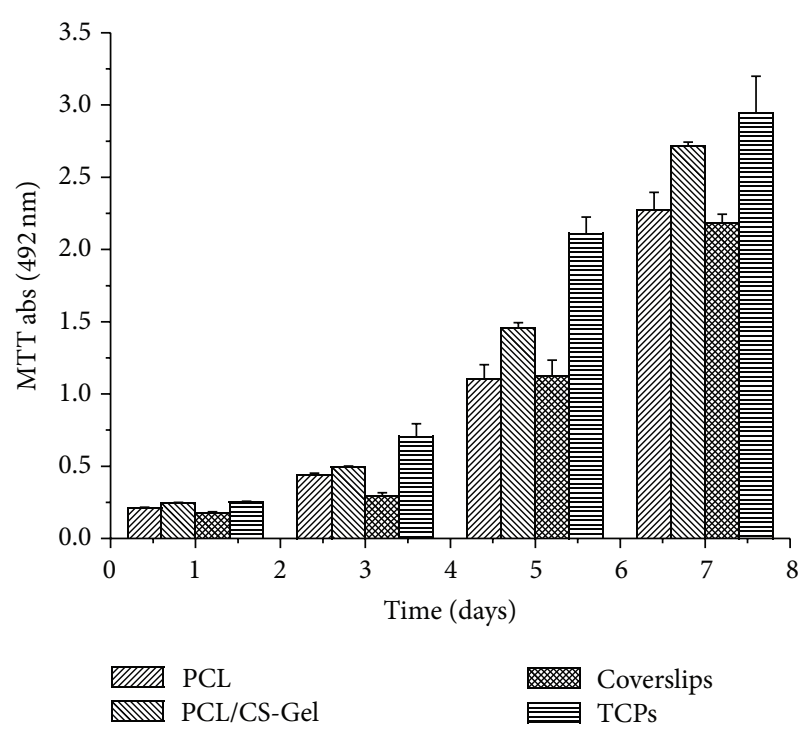

Figure 6: Proliferation of PIECs cultured on PCL, PCL/CS-Gel, cover slips, and TCPs.

\section{Conflict of Interests}

The authors declare that there is no conflict of interests.

\section{Acknowledgment}

This research was supported by a grant from The Education Department of Liaoning Province (Project no. 2012191).

\section{References}

[1] C. Xu, R. Inai, M. Kotaki, and S. Ramakrishna, "Electrospun nanofiber fabrication as synthetic extracellular matrix and its potential for vascular tissue engineering," Tissue Engineering, vol. 10, no. 7-8, pp. 1160-1168, 2004.

[2] J. Lee, G. Tae, Y. H. Kim, I. S. Park, S.-H. Kim, and S. H. Kim, "The effect of gelatin incorporation into electrospun poly $(L-$ lactide-co-e-caprolactone) fibers on mechanical properties and cytocompatibility," Biomaterials, vol. 29, no. 12, pp. 1872-1879, 2008.

[3] S. F. Badylak, "The extracellular matrix as a scaffold for tissue reconstruction," Seminars in Cell and Developmental Biology, vol. 13, no. 5, pp. 377-383, 2002.

[4] L. M. Bellan and H. G. Craighead, "Control of an electrospinning jet using electric focusing and jet-steering fields," Journal of Vacuum Science and Technology B, vol. 24, no. 6, pp. 3179-3183, 2006.

[5] H. M. Liou, L. R. Rau, C. C. Huang, M. R. Lu, and F. Y. Hsu, "Electrospun Hyaluronan-Gelatin nanofibrous matrix for nerve tissue engineering," Journal of Nanomaterials, vol. 2013, Article ID 613638, 9 pages, 2013.

[6] Z.-M. Huang, Y. Z. Zhang, S. Ramakrishna, and C. T. Lim, "Electrospinning and mechanical characterization of gelatin nanofibers," Polymer, vol. 45, no. 15, pp. 5361-5368, 2004.

[7] Y. Z. Zhang, B. Su, S. Ramakrishna, and C. T. Lim, "Chitosan nanofibers from an easily electrospinnable UHMWPEO-doped chitosan solution system," Biomacromolecules, vol. 9, no. 1, pp. 136-141, 2008.

[8] Y.-F. Qian, K.-H. Zhang, F. Chen, Q.-F. Ke, and X.-M. Mo, "Cross-linking of gelatin and chitosan complex nanofibers for tissue-engineering scaffolds," Journal of Biomaterials Science, Polymer Edition, vol. 22, no. 8, pp. 1099-1113, 2011.

[9] S. Gautam, A. K. Dinda, and N. C. Mishra, "Fabrication and characterization of PCL/gelatin composite nanofibrous scaffold for tissue engineering applications by electrospinning method," Materials Science and Engineering C, vol. 33, no. 3, pp. 12281235, 2013.

[10] M. I. Hassan, T. Sun, and N. Sultana, "Fabrication of nanohydroxyapatite/poly(caprolactone) composite microfibers using electrospinning technique for tissue engineering applications," Journal of Nanomaterials, vol. 2014, Article ID 209049, 7 pages, 2014.

[11] F. Roozbahani, N. Sultana, A. F. Ismail, and H. Noupavar, "Effect of chitosan alkali pretreatment on the preparation of electrospun PCL/Chitosan blend nanofibrous scaffolds for tissue engineering application," Journal of Nanomaterials, vol. 2013, Article ID 641502, 6 pages, 2013.

[12] E. Schnell, K. Klinkhammer, S. Balzer et al., "Guidance of glial cell migration and axonal growth on electrospun nanofibers of poly- $\varepsilon$-caprolactone and a collagen/poly- $\varepsilon$-caprolactone blend," Biomaterials, vol. 28, no. 19, pp. 3012-3025, 2007.

[13] C. H. Kim, M. S. Khil, H. Y. Kim, H. U. Lee, and K. Y. Jahng, "An improved hydrophilicity via electrospinning for enhanced cell attachment and proliferation," Journal of Biomedical Materials Research B Applied Biomaterials, vol. 78, no. 2, pp. 283-290, 2006.

[14] X. Zong, K. Kim, D. Fang, S. Ran, B. S. Hsiao, and B. $\mathrm{Chu}$, "Structure and process relationship of electrospun bioabsorbable nanofiber membranes," Polymer, vol. 43, no. 16, pp. 4403-4412, 2002.

[15] C. X. Zhang, X. Y. Yuan, L. L. Wu, Y. Han, and J. Sheng, "Study on morphology of electrospun poly(vinyl alcohol) mats," European Polymer Journal, vol. 41, no. 3, pp. 423-432, 2005.

[16] J. S. Choi, S. W. Lee, L. Jeong et al., "Effect of organosoluble salts on the nanofibrous structure of electrospun poly(3hydroxybutyrate-co-3-hydroxyvalerate)," International Journal of Biological Macromolecules, vol. 34, no. 4, pp. 249-256, 2004.

[17] T. Subbiah, G. S. Bhat, R. W. Tock, S. Parameswaran, and S. S. Ramkumar, "Electrospinning of nanofibers," Journal of Applied Polymer Science, vol. 96, no. 2, pp. 557-569, 2005.

[18] L. De Bartolo, S. Morelli, A. Bader, and E. Drioli, "The influence of polymeric membrane surface free energy on cell metabolic functions," Journal of Materials Science: Materials in Medicine, vol. 12, no. 10-12, pp. 959-963, 2001.

[19] K. E. Park, H. K. Kang, S. J. Lee, B.-M. Min, and W. H. Park, "Biomimetic nanofibrous scaffolds: preparation and characterization of PGA/chitin blend nanofibers," Biomacromolecules, vol. 7, no. 2, pp. 635-643, 2006.

[20] K. Zhang, H. Wang, C. Huang, Y. Su, X. Mo, and Y. Ikada, "Fabrication of silk fibroin blended P(LLA-CL) nanofibrous scaffolds for tissue engineering," Journal of Biomedical Materials Research A, vol. 93, no. 3, pp. 984-993, 2010. 

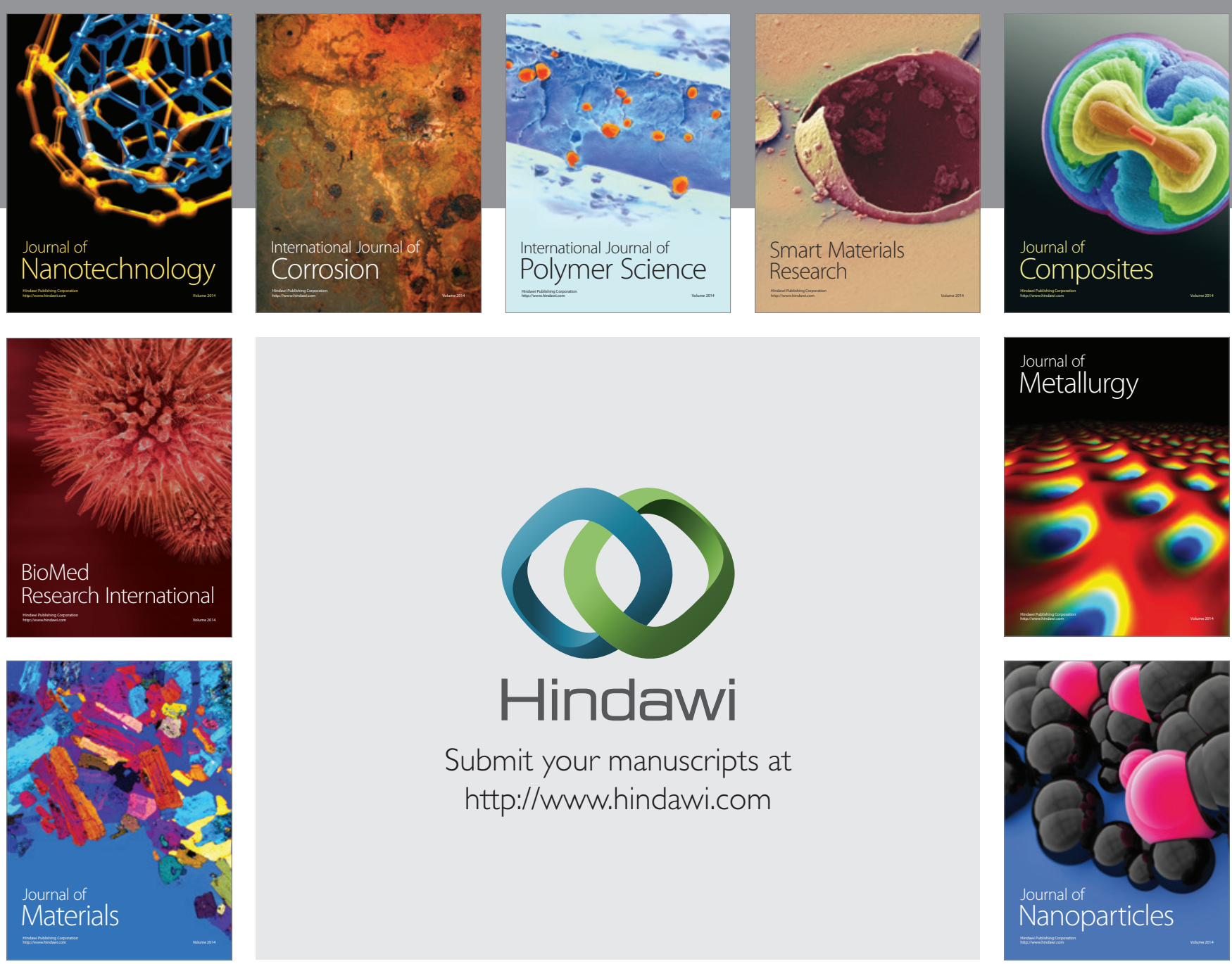

Submit your manuscripts at http://www.hindawi.com
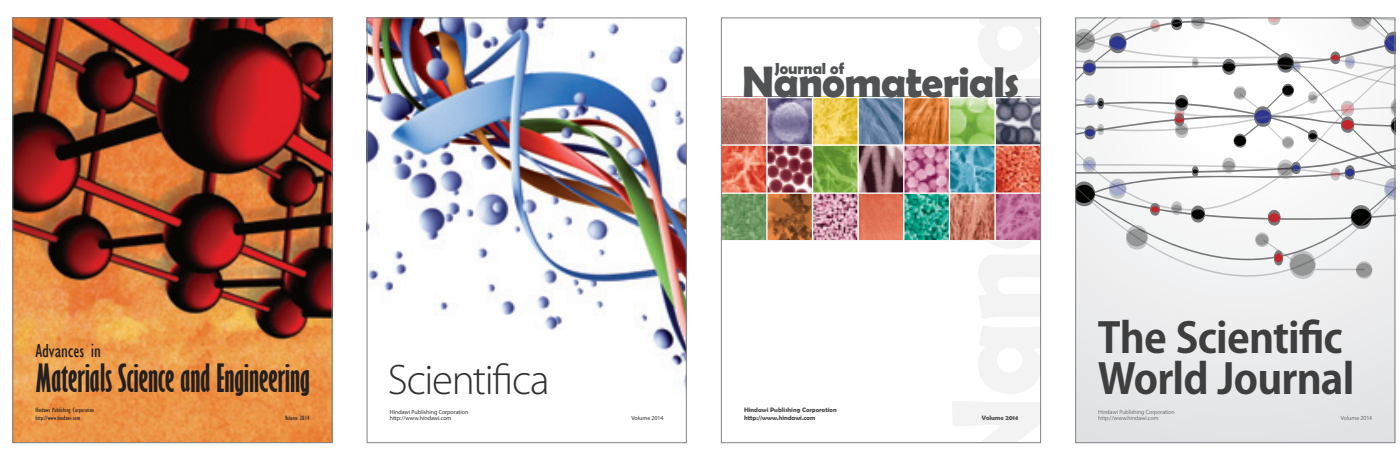

\section{The Scientific World Journal}
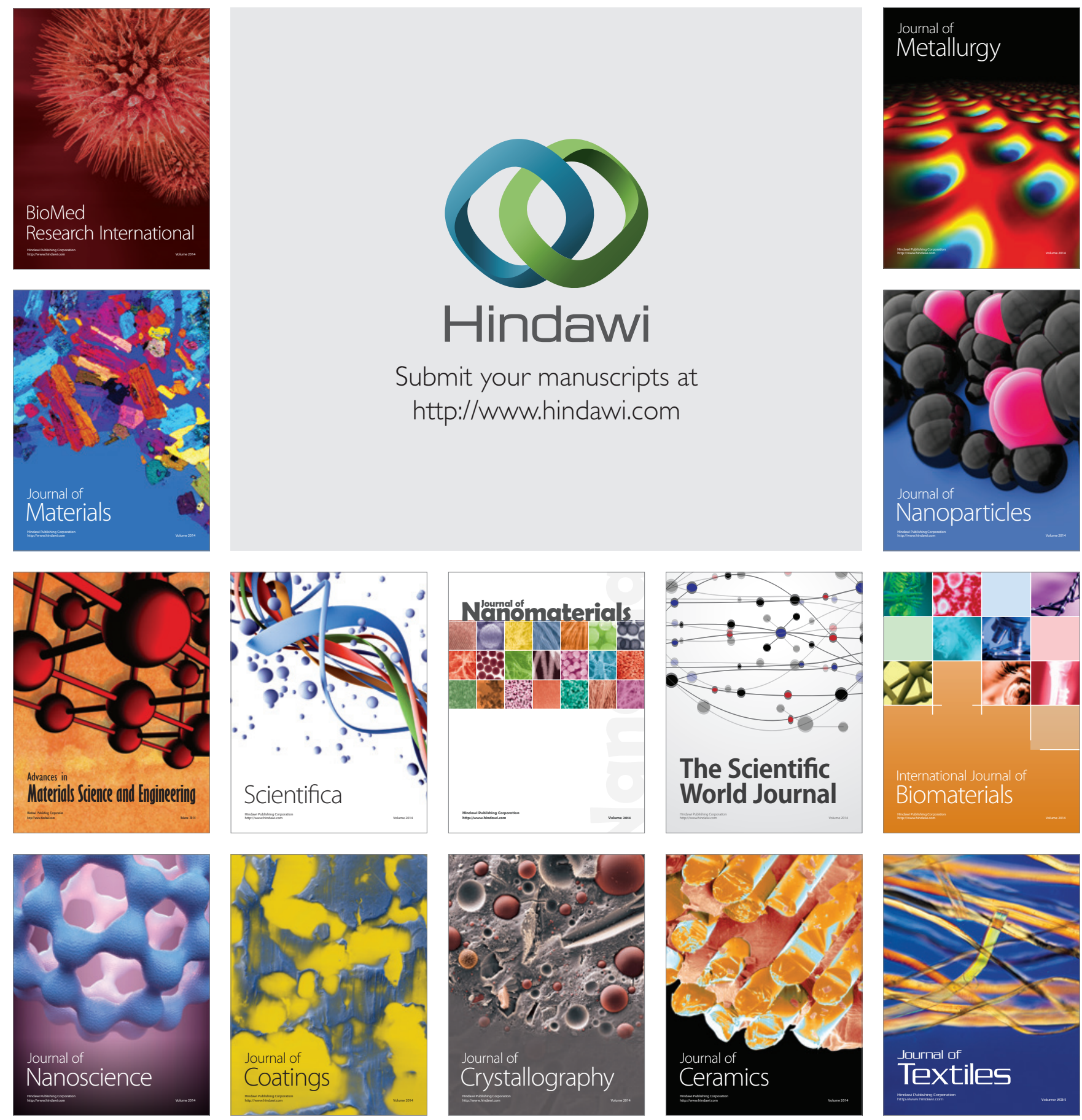